\title{
A NOTE ON THE SYMMETRIC RECURSIVE INVERSE EIGENVALUE PROBLEM
}

\author{
RAPHAEL LOEWY* AND VOLKER MEHRMANN ${ }^{\dagger}$
}

\begin{abstract}
In [1] the recursive inverse eigenvalue problem for matrices was introduced. In this paper we examine an open problem on the existence of symmetric positive semidefinite solutions that was posed there. We first give several counterexamples for the general case and then characterize under which further assumptions the conjecture is valid.
\end{abstract}

Keywords. Inverse eigenvalue problem, recursive solution, symmetric matrices, positive semidefinite matrices.

AMS subject classification. 15A29, 15A18, 15A48, 15A 57

Short title: Symmetric recursive inverse eigenvalue problem

1. Introduction. In [1] several classes of recursive inverse eigenvalue problems were introduced that construct matrices from eigenvalues and eigenvectors of leading principal submatrices. A simple application of such problems is the construction of Leontief models in economics, see e.g., [2], when a feasible model with $n-1$ inputs and $n-1$ outputs is extended (by adding an input and an output) to a larger feasible model with prescribed equilibrium point, see [1].

In this paper we discuss the particular case of the real symmetric recursive inverse eigenvalue problem, in the following denoted by $\operatorname{SRIEP}(n)$ which has the following form:

For given scalars $s_{1}, \ldots, s_{n} \in \mathbb{R}$ and real vectors

$$
r_{1}=\left[r_{1,1}\right], r_{2}=\left[\begin{array}{c}
r_{1,2} \\
r_{2,2}
\end{array}\right], \ldots, r_{n}=\left[\begin{array}{c}
r_{1, n} \\
\vdots \\
r_{n, n}
\end{array}\right]
$$

construct a symmetric matrix $A \in \mathbb{R}^{n, n}$ such that

$$
A[i] r_{i}=s_{i} r_{i} \quad i=1, \ldots, n,
$$

where $A[i]$ denotes the $i$-th leading principal submatrix of $A$.

We use the following notation. By o we denote the Hadamard (or elementwise) product of matrices. For an $n \times n$ matrix $A$ and increasing sequences $\alpha, \beta$ of elements in $\{1,2, \ldots, n\}, A[\alpha \mid \beta]$ denotes the submatrix of $A$ given by the row indices $\alpha$ and the column indices $\beta$. Futhermore, $A^{T}$ denotes the transpose of $A, A^{-T}$ the transpose of the inverse (if it exists), and $e_{i}$ denotes the i-th unit vector of appropriate dimension.

*Department of Mathematics, Technion, Haifa 32000, Israel. Email:loewy@techunix.technion.ac.il. Tel.: +972-4-829-4198 Fax: +972-4-832-4654. Support received through Technische Universität Berlin, Deutscher Akademischer Austauschdienst and the Fund for the Promotion of Research at the Technion.

†Institut für Mathematik, TU Berlin, D-10623 Berlin, FRG. Email: mehrmann@math.tuberlin.de. Tel.: +49-30-314-25736. Fax: +49-30-314-79706. Support received through DFG Research Center Mathematics for Key Technologies, TU Berlin 
The following matrices constructed from the data of the $\operatorname{SRIEP}(n)$ are used.

$$
R_{n}=\left[\begin{array}{cccc}
r_{1,1} & r_{1,2} & \ldots & r_{1, n} \\
0 & r_{2,2} & \ldots & r_{2, n} \\
\vdots & & & \vdots \\
0 & 0 & \ldots & r_{n, n}
\end{array}\right], S_{n}=\left[\begin{array}{ccccc}
s_{1} & s_{2} & s_{3} & \ldots & s_{n} \\
s_{2} & s_{2} & s_{3} & \ldots & s_{n} \\
s_{3} & s_{3} & s_{3} & \cdots & s_{n} \\
\vdots & & & & \vdots \\
s_{n} & s_{n} & \ldots & \cdots & s_{n}
\end{array}\right]
$$

In [1] the existence and uniqueness of solutions to $\operatorname{SRIEP}(n)$ is characterized, and in particular it is shown that if $R_{n}$ is invertible, i.e., all elements $r_{i, i}$ are nonzero, then the solution of $\operatorname{SRIEP}(n)$ exists, is unique and given by the formula

$$
A=R_{n}^{-T}\left(S_{n} \circ\left(R_{n}^{T} R_{n}\right)\right) R_{n}^{-1} .
$$

Thus the unique solution $A$ is positive definite [positive semidefinite] if and only if $S_{n} \circ\left(R_{n}^{T} R_{n}\right)$ is positive definite [positive semidefinite]. But if $R_{n}$ is singular and if a solution exists, then it is not unique, so a natural question to ask is whether there exists a positive definite [positive semidefinite] solution. It was also shown in [1] that any solution of $\operatorname{SRIEP}(n)$ must satisfy the matrix equation

$$
R_{n}^{T} A R_{n}=S_{n} \circ\left(R_{n}^{T} R_{n}\right)
$$

and hence it is clear that if there exists a positive definite [positive semidefinite] solution, then $S_{n} \circ\left(R_{n}^{T} R_{n}\right)$ has to be positive semidefinite. In [1] it was conjectured that the converse also holds, i.e.:

Let $n \geq 2$, and suppose that $S_{n} \circ\left(R_{n}^{T} R_{n}\right)$ is positive semidefinite [positive definite]. Then there exists a positive semidefinite [positive definite] solution for $\operatorname{SRIEP}(n)$.

In this paper we show that this conjecture is generally false. We give an example which shows that $\operatorname{SRIEP}(n)$ does not have to posess a solution at all if the assumption of the conjecture holds. Furthermore, the conjecture fails to hold even if we add the assumption that the problem has a solution, when $\operatorname{rank} S_{n} \circ\left(R_{n}^{T} R_{n}\right) \leq n-2$. We then prove that if a solution of $\operatorname{SRIEP}(n)$ exists, and $\operatorname{rank} S_{n} \circ\left(R_{n}^{T} R_{n}\right)>n-2$, then there exists a positive semidefinite [positive definite] solution for $\operatorname{SRIEP}(n)$.

2. Counterexamples. In this section we present several counterexamples that show that the conjecture in [1] as well as several obvious modifications do not hold.

EXAMPLE 2.1. Let $n=2, r_{1}=[1], r_{2}=\left[\begin{array}{l}1 \\ 0\end{array}\right], s_{1}=2, s_{2}=1$. Then

$$
R_{2}=\left[\begin{array}{ll}
1 & 1 \\
0 & 0
\end{array}\right], R_{2}^{T} R_{2}=\left[\begin{array}{ll}
1 & 1 \\
1 & 1
\end{array}\right], S_{2}=\left[\begin{array}{ll}
2 & 1 \\
1 & 1
\end{array}\right]
$$

and clearly $S_{2} \circ\left(R_{2}^{T} R_{2}\right)=\left[\begin{array}{ll}2 & 1 \\ 1 & 1\end{array}\right]$ is positive definite. Nevertheless, it is straightforward to check that there exists no matrix $A=\left[\begin{array}{ll}a_{11} & a_{12} \\ a_{21} & a_{22}\end{array}\right]$ such that $A[1] r_{1}=2 r_{1}$ and $A r_{2}=r_{2}$. Hence the conjecture is false as stated. In Example 2.1 the problem has no solution at all, so an immediate modification of the conjecture would be to require that the problem is solvable.

The next example shows that even with this modification the conjecture is false. 
EXAMPLE 2.2. Let $n=3, r_{1}=[1], r_{2}=\left[\begin{array}{l}2 \\ 0\end{array}\right], r_{3}=\left[\begin{array}{c}-1 \\ 1 \\ 1\end{array}\right], s_{1}=s_{2}=3$, and $s_{3}=9$. Then

$$
R_{3}=\left[\begin{array}{ccc}
1 & 2 & -1 \\
0 & 0 & 1 \\
0 & 0 & 1
\end{array}\right], R_{3}^{T} R_{3}=\left[\begin{array}{ccc}
1 & 2 & -1 \\
2 & 4 & -2 \\
-1 & -2 & 3
\end{array}\right], S_{3}=\left[\begin{array}{lll}
3 & 3 & 9 \\
3 & 3 & 9 \\
9 & 9 & 9
\end{array}\right]
$$

and clearly

$$
S_{3} \circ\left(R_{3}^{T} R_{3}\right)=\left[\begin{array}{ccc}
3 & 6 & -9 \\
6 & 12 & -18 \\
-9 & -18 & 27
\end{array}\right]
$$

is positive semidefinite and of rank 1 . The system of 6 equations for the elements of $A$ are

$$
\begin{aligned}
a_{1,1} & =3, \\
2 a_{1,1} & =6, \\
2 a_{1,2} & =0, \\
-a_{1,1}+a_{1,2}+a_{1,3} & =-9, \\
-a_{1,2}+a_{2,2}+a_{2,3} & =9, \\
-a_{1,3}+a_{2,3}+a_{3,3} & =9,
\end{aligned}
$$

which has the general solution

$$
A=\left[\begin{array}{ccc}
3 & 0 & -6 \\
0 & 9-a_{2,3} & a_{2,3} \\
-6 & a_{2,3} & 3-a_{2,3}
\end{array}\right],
$$

with $a_{2,3}$ to be chosen freely. But, since $\operatorname{det} A=3\left[\left(9-a_{2,3}\right)\left(3-a_{2,3}\right)-a_{2,3}^{2}\right]-36(9-$ $\left.a_{2,3}\right)=-245$ does not depend on $a_{2,3}$, clearly no positive semidefinite solution exists, although there exist symmetric solutions.

We can lift Example 2.2 to get counterexamples for all $n$, as long as rank $S_{n}$ 。 $\left(R_{n}^{T} R_{n}\right) \leq n-2$.

EXAMPLE 2.3. Let $n \geq 4, r_{1}=[1], r_{2}=\left[\begin{array}{l}2 \\ 0\end{array}\right], r_{3}=\left[\begin{array}{c}-1 \\ 1 \\ 1\end{array}\right], r_{i}=e_{i}$, for $i=4,5, \ldots, n$. Let, furthermore, $s_{1}=s_{2}=3, s_{3}=9$ and let $s_{i}, i=4,5 \ldots, n$ be any positive numbers. Then

$$
R_{n}=\left[\begin{array}{ccc|c}
1 & 2 & -1 & \\
0 & 0 & 1 & 0 \\
0 & 0 & 1 & \\
\hline & 0 & & I_{n-3}
\end{array}\right]
$$

and

$$
S_{n} \circ\left(R_{n}^{T} R_{n}\right)=\left[\begin{array}{ccc|ccc}
3 & 6 & -9 & & & \\
6 & 12 & -18 & & 0 & \\
-9 & -18 & 27 & & & \\
\hline & & & s_{4} & & \\
& 0 & & & \ddots & \\
& & & & & s_{n}
\end{array}\right]
$$


is positive semidefinite of rank $n-2$. The direct sum of $A$ from Example 2.2 and $I_{n-3}$ is a solution. If $B$ is any solution, then $B[3]$ necessarily is a solution for Example 2.2 and hence $B$ cannot be positive semidefinite.

These examples demonstrate that to prove the conjecture we have to require that the rank of $S_{n} \circ\left(R_{n}^{T} R_{n}\right)$ is at least $n-1$. In the next section we show that in this case the conjecture is true.

3. Main result. In this section we present our main result and prove the conjecture for the case that $\operatorname{rank}\left(S_{n} \circ\left(R_{n}^{T} R_{n}\right)\right) \geq n-1$.

THEOREM 3.1. Let matrices $R_{n}$ and $S_{n}$ be given such that $S_{n} \circ\left(R_{n}^{T} R_{n}\right)$ is positive semidefinite with $\operatorname{rank}\left(S_{n} \circ\left(R_{n}^{T} R_{n}\right)\right) \geq n-1$. If problem $\operatorname{SRIEP}(n)$ has a solution then it also has a positive semidefinite solution.

Proof. Suppose first that $\operatorname{rank}\left(S_{n} \circ\left(R_{n}^{T} R_{n}\right)\right)=n$, i.e., $S_{n} \circ\left(R_{n}^{T} R_{n}\right)$ is positive definite. Let $A$ be any solution of $\operatorname{SRIEP}(n)$. Then it has been shown in [1], that this solution must satisfy (1.3). This implies that $R_{n}$ is invertible and then it has been shown in [1] that the solution is unique, given by (1.2) and hence positive definite.

It remains to study the case that $\operatorname{rank}\left(S_{n} \circ\left(R_{n}^{T} R_{n}\right)\right)=n-1$. If $R_{n}$ is invertible, then again the solution $A$ is unique and given by (1.2) which is a positive semidefinite matrix of rank $n-1$. Hence, we may assume in the following that $R_{n}$ is singular.

Let $A$ be any particular solution of $\operatorname{SRIEP}(n)$. Then it follows from (1.3) that $\operatorname{rank} R_{n}=n-1$.

Using a sequence of elementary row and column operations [3], i.e., adding scalar multiples of one row (or column) to another, it follows that there exist invertible matrices $P, Q$ such that

$$
P R_{n} Q=\operatorname{diag}\left(\Sigma_{n-1}, 0\right),
$$

with $\Sigma_{n-1}$ of size $n-1 \times n-1$, diagonal and invertible. Actually we could achieve $\Sigma_{n-1}=I_{n-1}$, but we will use a different factorization below.

It follows from (1.3) that

$$
\left(Q^{T} R_{n}^{T} P^{T}\right)\left(P^{-T} A P^{-1}\right)\left(P R_{n} Q\right)=Q^{T}\left(S_{n} \circ\left(R_{n}^{T} R_{n}\right)\right) Q .
$$

Partition $\tilde{A}=P^{-T} A P^{-1}$ conformally with (3.1) as

$$
\tilde{A}=\left[\begin{array}{cc}
\tilde{A}_{1,1} & \tilde{A}_{1,2} \\
\tilde{A}_{1,2}^{T} & \tilde{A}_{2,2}
\end{array}\right] .
$$

Then it follows from (3.2) that

$$
Q^{T}\left(S_{n} \circ\left(R_{n}^{T} R_{n}\right)\right) Q=\left[\begin{array}{cc}
\Sigma_{n-1} \tilde{A}_{1,1} \Sigma_{n-1} & 0 \\
0 & 0
\end{array}\right]
$$

and hence, since the left side has rank $n-1$, we have that $\tilde{A}_{11}$ is positive definite.

Note that $\tilde{A}$ does not depend on $Q$, so we may choose $P$ and $Q$ so that the factorization (3.1) holds, while $P$ is as simple as possible. We now construct such a $P$ and, since $Q$ does not effect $\tilde{A}$, we do not record the column operations. Let

$$
Z=\left\{i \in\{1, \ldots, n\}: r_{i, i} \neq 0\right\}=\left\{i_{1}, \ldots, i_{m}\right\},
$$

where we assume that $1 \leq i_{1}<i_{2}<\ldots<i_{m} \leq n$. We call the entries $r_{i, i}$ with $i \in Z$ pivot elements of the first type. 
For every $i \in Z$, using elementary column operations, we can eliminate all offdiagonal elements in row $i$ and, since $R_{n}$ is upper triangular, this will not alter any of the diagonal elements. Hence the only nonzero element in row $i \in Z$ of the transformed matrix $\tilde{R}_{n}$ is the original diagonal element $r_{i, i}$. Moreover, $\tilde{R}_{n}=\left[\tilde{r}_{i, j}\right]$ is still upper triangular and of rank $n-1$.

Partition the set of indices $\tilde{Z}=\{1, \ldots, n\} \backslash Z$ into maximal disjoint subsets $Z_{1}, \ldots, Z_{k}$ of consecutive integers, representing the row numbers with vanishing diagonal elements $r_{j, j}$. For example, if the zero diagonal elements of $R_{n}$ are $r_{1,1}, r_{4,4}, r_{5,5}$, $r_{6,6}, r_{9,9}, r_{10,10}$ and $r_{14,14}$, then $Z_{1}=\{1\}, Z_{2}=\{4,5,6\}, Z_{3}=\{9,10\}$ and $Z_{4}=\{14\}$.

Consider now an arbitrary $Z_{j}$, where $1 \leq j \leq k$ and assume for simplicity that $Z_{j}=\{p, p+1, \ldots, p+q\}$, where $q \geq 0$. Then, since $\operatorname{rank} \tilde{R}_{n}=n-1$, it follows that if $q \geq 1$, then all entries $\tilde{r}_{l, l+1}, l=p, \ldots, p+q-1$ are nonzero. We call these entries pivot elements of the second type. Furthermore, for all the blocks associated with index sets $Z_{j}=\left\{p_{j}, \ldots, p_{j}+q_{j}\right\}, j=1, \ldots, k-1$, we have that there is at least the nonzero element $\tilde{r}_{p_{j}+q_{j}, s}$ in row $p_{j}+q_{j}$, where $s$ is the smallest element in $Z_{j+1}$. If this were not the case, then we would have that $\operatorname{rank} \tilde{R}_{n} \leq n-2$, a contradiction. We call the entries $\tilde{r}_{p_{j}+q_{j}, s}$ pivot elemnts of the third type.

Since there are no nonzero elements below the pivot elements of second type, we can perform further elementary column operations to eliminate more non pivot elements. Consider first $Z_{1}$ and eliminate (in the natural order) all the non-pivot elements in the in the rows associated using the pivots of second type. These operations do not affect any other rows associated with pivots of the second type or third type. Then we use the pivot element of the third type (if it exist) to annihilate the elements in its row, again without affecting any other rows. We proceed in the same way with the blocks associated with $Z_{2}, \ldots, Z_{k}$, again in the natural order.

Let $w$ denote the largest element of $Z_{k}$, and let $\hat{R}=\left[\hat{r}_{p, q}\right]$ denote the matrix obtained via this column operations applied to $\tilde{R}_{n}$. The matrix $\hat{R}_{n}$ has as nonzero elements all the pivot elements of first, second and third type, plus possibly some elements in row $w$. Since we have only used column operations, we have determined an invertible matrix $\hat{Q}$ such that $\hat{R}_{n}=R_{n} \hat{Q}$.

For the remainder of the proof we consider two cases.

Case 1: If $w=n$, then we have obtained (possibly after some additional permutation of columns) the desired form (3.1) with $P=I_{n}$ and hence $\tilde{A}=A$ and the submatrix $A[n-1]$ is positive definite. Since $r_{n, n}=0$, it follows that the homogeneous linear system corresponding to $\operatorname{SRIEP}(n)$ has the matrix $E_{n, n}=e_{n} e_{n}^{T}$ as solution. Thus all matrices of the form $\hat{A}(\alpha)=\alpha E_{n, n}+A$ with our particular solution $A$, are solutions and, since $A[n-1]$ is positive definite, choosing $\alpha>0$ sufficiently large, we obtain that $\hat{A}(\alpha)$ is positive definite.

Case 2: If $w<n$ then we need to perform elementary row operations using the pivots in rows $w+1, w+1, \ldots, n$ of $\hat{R}_{n}$ to annihilate the entries in positions $(w, w+1),(w, w+2), \ldots,(w, n)$ of $\hat{R}_{n}$. The corresponding pivot elements $r_{w+1, w+1}, r_{w+2, w+2}, \ldots, r_{n, n}$ are of first type.

Using Cramer's rule we can exactly determine the elements of $\hat{R}_{n}$ that we still have to eliminate, i.e.,

$$
\begin{aligned}
& \hat{r}_{w, w+1}=\operatorname{det} R_{n}[w \mid w+1] \\
& \hat{r}_{w, w+2}=-\frac{\operatorname{det} R_{n}[w, w+1 \mid w+1, w+2]}{r_{w+1, w+1}},
\end{aligned}
$$




$$
\begin{aligned}
\hat{r}_{w, w+3} & =\frac{\operatorname{det} R_{n}[w, w+1, w+2 \mid w+1, w+2, w+3]}{r_{w+1, w+1} r_{w+2, w+2}}, \\
& \vdots \\
\hat{r}_{w, n} & =(-1)^{n-w-1} \frac{\operatorname{det} R_{n}[w, w+1, \ldots, n-1 \mid w+1, w+2, \ldots, n]}{r_{w+1, w+1} r_{w+2, w+2} \cdots r_{n-1, n-1}} .
\end{aligned}
$$

Introducing the matrices of order $n-w+1$,

$$
C=\left[\begin{array}{cccccc}
0 & 1 & 0 & \ldots & \ldots & 0 \\
0 & 0 & 1 & 0 & \ldots & 0 \\
\vdots & \vdots & \ddots & \ddots & \ddots & \vdots \\
0 & 0 & \ldots & \ldots & 0 & 1 \\
1 & 0 & \ldots & \ldots & 0 & 0
\end{array}\right], M=I_{n-w+1}-e_{1}\left[\begin{array}{c}
0 \\
\frac{\hat{r}_{w, w+1}}{\hat{r}_{w+1, w+1}} \\
\ldots \\
\frac{\hat{r}_{w, n}}{\hat{r}_{n, n}}
\end{array}\right]^{T},
$$

and

$$
P_{1}=\left[\begin{array}{cc}
I_{w-1} & 0 \\
0 & C
\end{array}\right], P_{2}=\left[\begin{array}{cc}
I_{w-1} & 0 \\
0 & M
\end{array}\right],
$$

then with $P=P_{1} P_{2}$ we have obtained invertible matrices $P, Q$ such that (3.1) holds. Recall that for $\tilde{A}=P^{-T} A P^{-1}$ we have $\tilde{A}[n-1]$ is positive definite.

As in Case 1, we show that there exists a rank 1 positive semidefinite solution $A_{0}$ of the homogeneous linear system corresponding to $\operatorname{SRIEP}(n)$ and a scalar $\alpha>0$ such that that $\tilde{A}+\alpha P^{-T} A_{0} P^{-1}=P^{-T}\left(A+\alpha A_{0}\right) P^{-1}$ is a positive definite solution of $\operatorname{SRIEP}(n)$.

Let

$$
\begin{aligned}
& \tilde{z}^{T}=\left[1, \quad \frac{-\operatorname{det} R_{n}[w \mid w+1]}{\hat{r}_{w+1, w+1}}, \quad \frac{\operatorname{det} R_{n}[w, w+1 \mid w+1, w+2]}{\hat{r}_{w+1, w+1} \hat{r}_{w+2, w+2}}, \quad \ldots\right. \\
& \left.\ldots, \quad(-1)^{n-w} \frac{\operatorname{det} R_{n}[w, w+1, \ldots, n-1 \mid w+1, w+2, \ldots, n]}{\hat{r}_{w+1, w+1} \cdots \hat{r}_{n, n}}\right],
\end{aligned}
$$

and if $A_{0}=z z^{T}$, where $z^{T}=\left[\begin{array}{llll}0 & \ldots & 0 & \tilde{z}^{T}\end{array}\right]$, then $A_{0}$ satisfies the homogeneous system $A_{0}[i] r_{i}=0$ for $i=1,2, \ldots, n$. To show this it suffices to prove that $z^{T} R_{n} e_{i}=$ 0 , for $i=1, \ldots, n$. This is clear for $i=1, \ldots, w-1$ because of the zeros in $z$ and for $i=w$, since $r_{w, w}=0$. To prove this for $i=w+1, \ldots, n$, we have to show that

$$
\tilde{z}^{T} R_{n}[w, w+1, \ldots, n \mid w+1, w+2, \ldots, n]=0,
$$

but this is exactly how we have constructed $\tilde{z}$ and follows from Cramer's rule.

By construction we also have that $z^{T} P^{-1}=e_{n}^{T}$ and hence

$$
P^{-T} A_{0} P^{-1}=P^{-T} z z^{T} P^{-1}=e_{n} e_{n}^{T}=E_{n, n} .
$$

The same argument as in Case 1 gives the existence of a positive definite solution. $\quad$ G

The interesting case in the proof of Theorem 3.1 is when $\operatorname{rank} R_{n}=n-1$. In this case we needed to add a particular solution of the homogeneous system corresponding to $\operatorname{SRIEP}(n)$ in order to get a positive definite solution.

Thus it is intersting to study the homogeneous system in slightly more detail.

TheOREM 3.2. Let $n \geq 2$ and consider the homogeneous system

$$
A[i] r_{i}=0, i=1,2, \ldots, n
$$


associated with $\operatorname{SRIEP}(n)$, and suppose that $\operatorname{rank} R_{n}=n-1$. Let $w$ be the largest integer such that $r_{i, i}=0$. Then the general solution of (3.3) has dimension $w$ if $r_{w}=0$ and dimension $w-1$ if $r_{w} \neq 0$. Moreover, for any solution $A$ of (3.3) we have $A[w-1]=0$. Furthermore, if $r_{w}=0$, then the elements $a_{1, w}, \ldots, a_{w, w}$ can be chosen to be the free variables in the solution of (3.3). If $r_{w} \neq 0$ and $s$ is the smallest integer such that $r_{s, w} \neq 0$, then $a_{1, w}, a_{2, w}, \ldots a_{s-1, w}, a_{s+1, w}, \ldots, a_{w, w}$ can be chosen to be the free variables in the solution of (3.3). Here, if $w=1$, we mean that $a_{1,1}$ is the only free variable.

Proof. The proof is by induction on $n$. The case $n=2$ is trivial. Suppose first that $w<n$. Consider the subsystem of (3.3) given by

$$
A[i] r_{i}=0, i=1,2, \ldots, w
$$

and apply the induction hypothesis. Since all diagonal entries $r_{w+1, w+1}, \ldots, r_{n, n}$ are nonzero, the system $A[w+1] r_{w+1}=0$ will determine $a_{1, w+1}, \ldots, a_{w+1, w+1}$ uniquely in terms of the free variables of (3.4). Continuing in this way with the equations $A[w+j] r_{w+j}=0, j=2, \ldots, n-w$, we determine all the remaining entries of $A$ in terms of the free variables of (3.4).

So we may assume that $w=n$, i.e., $r_{n, n}=0$, and therefore the whole last row of $R_{n}$ is zero. For $i=1,2, \ldots, n-1$ let

$$
a_{(i)}=\left[a_{i, 1}, a_{i, 2}, \ldots, a_{i, n-1}\right]
$$

and let $\hat{r}_{j}$ denote the vector obtained by deleting the last entry of $r_{j}, j=1,2, \ldots, n$. Since rank $R_{n}=n-1$, the first $n-1$ rows of $R_{n}$ are linearly independent, implying that $\hat{r}_{1}, \hat{r}_{2}, \ldots, \hat{r}_{n}$ span $\mathbb{R}^{n-1}$. Considering the row vector $a_{(1)}$, it follows from (3.3) that $a_{(1)} \hat{r}_{j}=0$ for $j=1,2, \ldots, n$ and hence it follows that $a_{(1)}=0$, in particular $a_{1,2}=a_{2,1}=0$. Then for $a_{(2)}=\left[0, a_{2,2}, \ldots, a_{2, n-1}\right]$ we have $a_{(2)} \hat{r}_{1}=0$, since $a_{2,1}=0$ and $a_{(2)} \hat{r}_{j}=0$ for $j=2, \ldots, n$ by $(3.3)$, and hence $a_{(2)}=0$. In particular we have $a_{1,3}=a_{3,1}=a_{2,3}=a_{3,2}=0$. Proceeding inductively, we obtain in a similar way that $a_{(3)}=a_{(4)}=\ldots=a_{(n-1)}=0$ and hence $A[n-1]=0$. It remains to consider $A r_{n}=0$. If $r_{n}=0$ this is automatically satisfied and hence $a_{1, n}, a_{2, n}, \ldots, a_{n, n}$ are the free variables. Otherwise, if $r_{n} \neq 0$ then there is a single linear equation

$$
r_{1, n} a_{1, n}+r_{2, n} a_{2, n}+\ldots+r_{n, n-1} a_{n-1, n}=0
$$

for the free variables. This concludes the proof.

We have given conditions so that there exists a positive semidefinite [positive definite] solution to $\operatorname{SRIEP}(n)$ that depend just on the fact that $S_{n} \circ\left(R_{n}^{T} R_{n}\right)$ is positive semidefinite [positive definite], but no use of the special structure of the matrix $S_{n}$ is made. Some sufficient conditions that use just inequalities between the $s_{j}$ are given in [1]. For example it is shown there that if $R_{n}$ is invertible and $s_{1}>s_{2}>\ldots>s_{n} \geq 0$ then the unique solution of $\operatorname{SRIEP}(n)$ is positive semidefinite and if $s_{n}>0$ then the unique solution is positive definite. But these inequalities are not necessary to have a positive semidefinite solution.

4. Conclusion. We have presented counterexamples to a conjecture posed in [1] and then have presented conditions under which the conjecture holds.

\section{REFERENCES}


[1] M. Arav, D. Hershkowitz, V. Mehrmann, and H. Schneider, The Recursive inverse eigenvalue problem. SIAM J. Matrix Anal. and Appl. 22:392-412, 2000.

[2] A. Berman and R. Plemmons, Nonnegative Matrices in the Mathematical Sciences, SIAM, Philadelphia, 1994.

[3] G.H. Golub and C.F. Van Loan, Matrix Computations, 3rd Ed., Johns Hopkins Univ. Press, 1996.

[4] R.A. Horn and C.R. Johnson, Matrix Analysis, Cambridge University Press, Cambridge, 1985. 